\title{
Sketchy With a Chance of Adoption: Can Sketch-Based Telemetry Be Ready for Prime Time?
}

\author{
Zaoxing Liu ${ }^{1,2}$, Hun Namkung ${ }^{1}$, Anup Agarwal ${ }^{1}$, Antonis Manousis ${ }^{1}$, \\ Peter Steenkiste ${ }^{1}$, Srinivasan Seshan ${ }^{1}$, Vyas Sekar ${ }^{1}$ \\ ${ }^{1}$ Carnegie Mellon University, ${ }^{2}$ Boston University
}

\begin{abstract}
Sketching algorithms or sketches have emerged as a promising alternative to the traditional packet sampling-based network telemetry solutions. At a high level, they are attractive because of their high resource efficiency and accuracy guarantees. While there have been significant recent advances in various aspects of sketching for networking tasks, many fundamental challenges remain unsolved that are likely stumbling blocks for adoption. Our contribution in this paper is in identifying and formulating these research challenges across the ecosystem encompassing network operators, platform vendors/developers, and algorithm designers. We hope that these serve as a necessary fillip for the community to enable the broader adoption of sketch-based telemetry.
\end{abstract}

\section{INTRODUCTION}

At the core of managing networks, network telemetry plays a crucial role in understanding what is happening in the network and informing management decisions. For example, to improve cloud security, telemetry enables operators to detect network anomalies and attacks in a timely fashion. Similarly, in order to optimize traffic engineering and ensure that service-level agreements (SLAs) for applications are met, operators commonly rely on telemetry to monitor network flow distributions. Traditionally, flow-based telemetry is done via offline analysis or some form of packet or flow sampling (e.g., NetFlow [22] and sFlow [56]). However, given the need for timely results using constrained compute/memory resources, offline analysis is not a practical option. Moreover, sampling only provides coarse-grained flow size distributions, and cannot provide accurate results for more finegrained key telemetry tasks such as entropy estimation, distinct count, and change detection [28, 29, 42].

To address the drawbacks of sampling approaches, sketching algorithms (or sketches for short) have been extensively studied in recent years (e.g., [13, 14, 21, 25, 36, 37, 62, 39. 45, 59, 53, 44, 60, 50, 42, 46, 52]). In light of increasing network traffic and ever-evolving application dynamics, sketches have emerged as a promising solution for real-time network telemetry.

This paper is a reflection on the current state of sketchbased telemetry to examine not just what sketch-based sys-

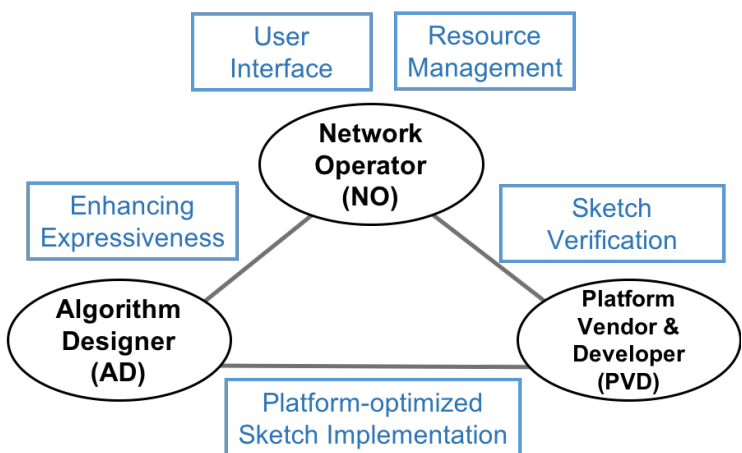

Figure 1: Overview of the problems from the stakeholders in sketch-based telemetry.

tems can do but what should be done to enable broader adoption. To this end, we look at the state of the sketch-based telemetry ecosystem from the perspective of three key stakeholders in Figure 1. (1) Network Operators (NO) who are the users/consumers of telemetry capabilities; (2) Algorithm Designers $(A D)$ who design and analyze sketching algorithms; and (3) Platform Vendors and Developers (PVD) who provide hardware/software primitives and APIs in various platforms (e.g., Intel DPDK [4], Barefoot Tofino [2], Broadcom Trident [3], Mellanox [5], among others) and use these APIs to implement sketch-based functions.

By taking this ecosystem-level view, we identify four areas of gaps between stakeholder and interaction requirements and existing research (blue boxes in Figure 1):

- NO-Centric: Most existing efforts assume operators have extensive knowledge about the algorithms and their underlying data structures, which is not realistic. There are few, if any, efforts to help operators translate high-level intents into sketches. This requires both high-level interfaces as well as precise resource management. While NO's intents may involve different sketches and devices, current solutions (e.g., [45, 59, 37, 48]) do not consider the composition of multiple types of sketches and the heterogeneity of network devices.

- Between NO/AD: Prior theoretical work in sketching algorithms covers many common telemetry tasks, and more recent work on general sketches can cover a broad portfolio of tasks [45]. Despite these advances, many common 
NO intents fall outside the scope of the literature. For instance, for attack detection, operators are interested in obtaining statistics from not only one dimension of data (e.g., SrcIP) but multiple dimensions (e.g., any subset of the combinations in 5-tuple). Conversely, we find that the theory community has many rich capabilities and streaming models (e.g., turnstile [43, 55], sliding-window [27, 18, 19], and distributed functional monitoring [24, 57]) that are yet to find practical adoption in networking.

- Between AD/PVD: While sketching algorithms are theoretically lightweight, existing algorithms may not be efficiently realizable across diverse platforms as highlighted by recent efforts [36, 44, 59, 53, 58]. Similarly, while existing languages and APIs [1, 16, 6] are sufficiently expressive to specify different sketch algorithms, naïve implementations are often resource intensive, thus nullifying any potential benefits [36, 44]. This suggests the need for new sketch-centric APIs, language support, and best practices.

- Between NO/PVD: Given that the success of the operator's policies depends crucially on how accurately telemetry reflects current network conditions, verifying the practical accuracy and correctness of sketches at post-deployment is a major priority for the NO. In addition, while platform vendors have designed and delivered trusted hardware capabilities (e.g., Intel SGX [38], AMD SEV [10], and ARM TrustZone [12]) to ensure the integrity of the program running on the device, the integrity of sketch-based telemetry logic has yet to be protected.

Our contribution in this paper is to identify and formulate challenges that need to be addressed to enable sketchbased telemetry to be more widely adopted. While this list of challenges is by no means exhaustive, our goal is to start the conversation regarding the ecosystem's missing pieces. We hope that our work will inspire the community to tackle these as-yet-unsolved issues, eventually enabling the practical adoption of sketch-based telemetry.

\section{BACKGROUND}

In this section, we first provide some background on sketches and their use in network telemetry. We then introduce the key stakeholders in sketch-based telemetry to set the context for the research challenges.

\subsection{Sketching Algorithms}

Sketching algorithms (sketches) can process data streams accurately and efficiently in an online fashion. Sketches are attractive for network monitoring precisely because they typically require small memory footprints to estimate traffic statistics with provable accuracy guarantees. In addition to network telemetry [21, 25, 47, 50, 45, 60, 59, 36, 37], sketchbased approaches have also been applied in databases [7, 31], streaming analytics [11], and machine learning [33, 40, 41].

Sketches draw on rich theoretical foundations starting from the foundational "AMS" paper [8]. At a high level, the prob- lem they address is as follows: Given an input stream of $<$ key, value > pairs (e.g., <5-tuple, packet size> pairs in network traffic), a sketching algorithm is allowed to make a single pass over the data stream to compute statistics while using sub-linear (usually poly-logarithmic) memory space compared to the total size of the dataset and the number of distinct keys. When processing each item in the stream, a sketch typically maintains a table of counters in the main memory and computes multiple independent hashes to update a small random set of counters in the table. These algorithms are backed by rigorous theoretical analysis on bounded accuracy-memory tradeoffs for arbitrary workload patterns.

Sketch-based network telemetry. Sketches are useful approaches for key network telemetry tasks, such as (1) HeavyHitter detection to discover large flows [25, 21, 47, 53, 45, 59]; (2) Entropy Estimation to analyze traffic distributions for anomaly detection [23, 45, 50]; (3) Change Detection to identify significant traffic shifts over time [42, 60, 45]; (4) Cardinality Estimates to detect the number of distinct items/flows in the network traffic [13, 30, 45, 36]; (5) Performance Monitoring to identify flows with high packet loss, large latency, and high out-of-order or retransmitted packets [46]; (6) Superspreader Detection to identify sources that contact many different destinations [60], among others.

\subsection{Stakeholders for Telemetry Deployment}

We identify three key players in the ecosystem that drive and influence the adoption of the above sketch-based telemetry.

Network operator: Network operators rely on real time telemetry to make timely decisions that ensure network reliability, performance, and security. To this end, they may want network-wide information such as global heavy hitter flows, distinct flows, and entropy changes on various traffic distributions. Ideally, network operators want to express highlevel telemetry objectives without having to worry about lowlevel algorithm and implementation details about sketches.

\section{Q1: Return 5-tuple 0.005-heavy hitters from all flows} $\mathrm{FlowKey}=$ (SrcIP, SrcPort, DstIP, DstPort, Proto)
$\mathrm{C}=$ "Select HeavyHitter $(\mathrm{P}$. FlowKey, 0.05) From *" return $\mathrm{C}$

\section{Q2: Return distinct DstIP count that a host connects to}

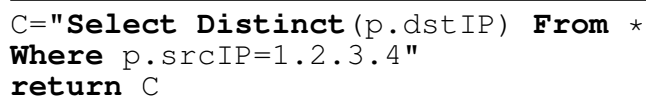

\section{Figure 2: Examples of envisioned telemetry queries.}

For example, operators may specify queries like Q1 and Q2 depicted in Figure 2. A telemetry system should provide an interface to write queries, identify if the queries can be supported by existing primitives, and distribute the monitoring responsibilities efficiently across a network. If better or new sketches are needed, the telemetry system must pass these information to algorithm designers described below. 


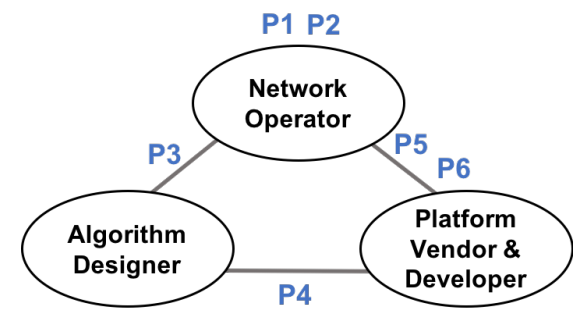

Figure 3: Open problems between stakeholders.

Algorithm designers: We envision an active community of algorithm designers developing new sketching algorithms to estimate different telemetry metrics. They would like to understand the requirements of network operators to design improved or new sketching algorithms for needed metrics. In practice, however, it requires significant efforts to translate theoretical algorithms into optimized implementations on diverse platforms. As richer primitives need to be designed and new platforms (e.g., Barefoot Tofino [2] and Multi-engine SoC SmartNIC [6]) emerge, algorithm designers increasingly find themselves in need of a mature sketch-based framework allowing them to develop and evaluate algorithmic tools along with platform vendors/developers described below.

Platform vendors and developers: Platform vendors offer specialized capabilities that implement and optimize sketches on various hardware and software platforms. For instance, we have already seen programmable switches, SmartNIC, FPGA, and software switches established in today's networks, and we envision future deployments with richer and more diverse platform capabilities. Ideally, platform vendors should provide primitives for these developers to optimally support sketch-based telemetry. However, recent work suggests it is non-trivial to efficiently implement sketches [44, 53]. In this respect, we envision the need for these two stakeholders to jointly contribute their domain expertise to achieve optimized sketch implementations.

\section{RESEARCH CHALLENGES}

Next, we formulate a broad (but non-exhaustive) list of open research problems P1... P6 and some of their extensions for a sketch-based telemetry ecosystem. As depicted in Figure 3, we conceptually cluster these challenges according to each stakeholder's needs and considerations.

Preliminaries: We introduce some terms and notations to formulate the problems (summarized in Table 1 ).

- The constants represent the inputs to the telemetry system. Specifically, network operators can define their telemetry needs by a list of input constants: (1) Queries $\mathcal{Q}$ consisting of a set of $k$ (potentially infinite) query definitions $\left\{q_{1}, \ldots, q_{k}\right\} ;$ (2) Requirements $\mathcal{R}_{\mathcal{A}}=\left\{r_{a}{ }^{1}, \ldots, r_{a}{ }^{k}\right\}$ defining a set of accuracy requirements (e.g., accuracy target 95\% with 0.99 confidence) for queries $\left\{q_{1}, \ldots, q_{k}\right\}$ and similarly $\mathcal{R}_{\mathcal{P}}=\left\{r_{p}{ }^{1}, \ldots, r_{p}{ }^{k}\right\}$ as the packet rate requirements; (3) Network characteristics including topology information $\mathcal{T}$, device information with resource capabilities $\mathcal{D}$, and traffic workload characteristics $\mathcal{W}_{\nabla}$.

\begin{tabular}{|c|c|}
\hline Constants & Definition \\
\hline $\mathcal{Q}$ & Set of telemetry queries \\
\hline $\mathcal{R}_{\mathcal{A}}$ & $\begin{array}{l}\text { Set of accuracy requirements, } \\
\text { e.g., accuracy target and confidence level }\end{array}$ \\
\hline $\mathcal{R}_{\mathcal{P}}$ & Set of performance requirements, e.g., packet rate \\
\hline $\mathcal{T}$ & Topology information, e.g., links and devices \\
\hline $\mathcal{D}$ & $\begin{array}{l}\text { Set of device instances with resource constraints, } \\
\text { e.g., SmartNIC w/ } 4 \text { engines and 10MB SRAM }\end{array}$ \\
\hline $\mathcal{W}_{\nabla}$ & Traffic workload characteristics, e.g., distribution \\
\hline Variables & Definition \\
\hline$S$ & Set of sketch definitions with configurations \\
\hline$r_{s, d}$ & Resource config. for sketch s on device $d$ \\
\hline$l_{s, d}$ & Processing latency for sketch s on device $d$ \\
\hline$c_{s, d}$ & Implementation of sketch $s$ on device $d$ \\
\hline$c_{d}$ & Implementation of all sketches on device $d$ \\
\hline$r_{d}$ & Actual resource usage of device $d$ from $c_{d}$ \\
\hline$l_{d}$ & Actual processing latency of device $d$ from $c_{d}$ \\
\hline
\end{tabular}

Table 1: Summary of notations in problem definitions.

- The variables are the notations for the intermediate or final outputs of the telemetry system: (1) $S$ is a set of sketch definitions with appropriate memory and flow-key/OD-pair configurations (e.g., a Count-Min sketch tracking 5-tuple flows with $5 \times 2048$ 32-bit counters); (2) $r_{s, d}$ is the resource configuration of sketch instance $s$ on device $d$ (e.g., assigning $200 \mathrm{~KB}$ and 2 cores for $s$ on CPU) and $l_{s, d}$ is the processing latency of $s$ on $d$ (e.g., $1 \mu s$ on CPU); (3) $c_{s, d}$ is the implementation (binary code) of sketch instance $s$ on device $d$. When there are multiple sketch instances in $d, c_{d}$ represents the implementation of all instances combined; (4) $r_{d}$ is the actual resource usage of $c_{d}$ and $l_{d}$ is the actual processing latency of $c_{d}$.

\subsection{Network Operator-Centric}

$\overline{\text { Problem 1: [Query Language] Is there a high-level declara- }}$ tive language that can precisely define sketch-based telemetry queries $\mathcal{Q}$ ?

Traditionally, sketch-based telemetry is designed under a narrow scope in the queries it supports. Specifically, existing frameworks are either designed to support one type of queries [31] or assume that the operators determine at query time the appropriate (available) sketch for each query. For example, to detect Superspreaders (i.e., SrcIPs that connect to many distinct DstIPs), the operators need to make a choice between Count-Min + HLL and CountSketch + UnivMon whereas to conduct change detection they need to choose between K-ary and Count-Min. As a result, developing a unified front-end for such telemetry systems was, to the best of our knowledge, never seen as a key design priority. Specifically, the operators should be able to conceptually describe the characteristics of a query to execute (e.g., type of metrics, appropriate aggregation of data, accuracy constraints) without explicitly specifying the execution mechanism.

Existing efforts have proposed several query languages for network telemetry [34, 61, 49], streaming database [20, 26], and traffic analysis [15]. These efforts are self-contained for their systems but may not be an ideal fit for sketch-based 
telemetry. Specifically, they did not consider sketches as their primitives and overly complicate the query definitions for sketches. For instance, Sonata [34] can specify the detailed packet-level queries with dataflow operators (e.g., map, filter, reduce) but it is unclear how to describe sketches, and NetQRE [61] extends from quantitative regular expressions [9] to define flow-level and application-level statistics and polices. In addition, the telemetry tool Marple [49] is designed to support a particular set of performance metrics only. Similarly, streaming databases such as Gigascope [26] support continuous queries over packet headers or counts via a SQLalike language but do not support other metrics such as network performance and traffic patterns.

Problem 2: [Resource Optimization] Given a set of queries $\mathcal{Q}$ with accuracy requirements $\mathcal{R}_{\mathcal{A}}$ and performance requirements $\mathcal{R}_{\mathcal{P}}$, traffic workload characteristics $\mathcal{W}_{\nabla}$, topology $\mathcal{T}$, and device instances $\mathcal{D}$, generate resource configuration $r_{s, d} \forall s, d$ within a time budget such that $\sum_{s} \sum_{d} r_{s, d}$ is minimized and $\forall s \in S$ meets $\mathcal{R}_{\mathcal{A}}$ and $\mathcal{R}_{\mathcal{P}}$

Given a set of queries $\mathcal{Q}$, each with associated accuracy and performance requirements, traffic workload characteristics and a network topology, the operator's high-level goal is to deploy appropriate sketches across the deployment such that SLAs are met while minimizing overall resource usage. The operator ideally wants to view their deployment under as "one-big-switch" without worrying about manually distributing sketches across the various devices in the deployment to ensure appropriate correctness and coverage. However, realizing this conceptual goal, requires addressing a number of sub-challenges which we introduce now and discuss in more detail in the following subsections:

- Problem 3: Translate each $q \in \mathcal{Q}$ to appropriate sketch definitions with conservative (traffic-oblivious) memory configurations $S$ to meet accuracy requirements $\mathcal{R}_{\mathcal{A}}$.

- Problem 4: Given a heterogeneous network deployment, develop optimal device-specific sketch implementations, given sketch definitions and configurations $s \in S$.

- Problem 5: Given traffic workload characteristics $\mathcal{W}_{\nabla}$, optimize each sketch's memory configuration to provide better memory-accuracy tradeoff and further reduce resource usage.

- Problem 6: Once sketches are deployed on device $d$, verify their correctness to ensure the expected accuracy requirements $\mathcal{R}_{\mathcal{A}}$ are met.

While prior work presented an early version of a networkwide solution [45], it does not take traffic workload characteristics, different types of sketches, and the heterogeneity of the devices into account, and can converge to a suboptimal or even infeasible sketch placement and resource allocation. Figure 4 shows a simple scenario where networkwide UnivMon does not optimally place three Count-Min sketch instances in a topology of three programmable devices. Specifically, in the example, the operator wants to know the 5-tuple heavy hitters over traffic between devices $A$ and $C$ (CM1) and the heavy hitters over traffic between devices B and C separately for (SrcIP, SrcPort) and (DstIP, DstPort) flow keys (CM2 and CM3). Resource optimization approaches will decide which sketch will be placed on which device while being aware of the resources required for these sketches given different performance requirements for different devices: (1) UnivMon, which is unaware of the interaction between performance requirements and resource usage, tries to balance memory usage by placing a sketch on each device. This results in placing a sketch on device $A$ which sees $20 \mathrm{Mpps}$ traffic. In order to accommodate a sketch and support this forwarding rate, device $A$ requires 4 cores. (2) A better strategy shifts telemetry load towards device $B \square$ which sees less traffic and can accommodate 2 sketches while meeting the $10 \mathrm{Mpps}$ requirement. Device $A$ in this strategy does not maintain a sketch and only needs 2 cores to maintain $20 \mathrm{Mpps}$ traffic forwarding. Note: Device $C$ 's compute resources are the same in both strategies and hence are not shown.

Extension: [Maximum Performance] Given a set of queries $\mathcal{Q}$ with requirements $\mathcal{R}_{\mathcal{A}}$, topology information $\mathcal{T}$, and devices $\mathcal{D}$, output resource configuration $r_{s, d}$ for all $s, d$ such that $\sum_{s} \sum_{d} l_{s, d}$ is minimized and $\forall s \in S$ meets $\mathcal{R}_{\mathcal{A}}$

This extension aims at providing optimized network-wide sketch placement and resource allocation that meets the device resource constraints and minimizes total packet processing overhead. In this optimization, we aim at deploying a telemetry solution to handle the largest possible volume of traffic for given queries, which potentially offers us the ability to monitor bursty traffic. Meanwhile, this type of optimization is useful for operators to control the maximum volume of traffic that goes into the telemetry infrastructure.

\subsection{Network Operator \& Algorithm Designer}

Problem 3: [Queries to Sketch Definitions] Design a compiler that translates queries $\mathcal{Q}$ into sketch definitions and configurations $S$ that meet accuracy requirements $\mathcal{R}_{\mathcal{A}}$

Here, our focus is on translating telemetry queries into a set of practical sketch definitions with memory configurations satisfying the accuracy requirements from the queries, irrespective of traffic workload characteristics and hardware platforms. This is possible because the accuracy guarantees of sketches are hardware agnostic and only depend on the memory configuration. Thus, one can potentially leverage the theoretical analysis from algorithm designers to provide traffic-oblivious sketch memory configurations. For example, if a query specifies a heavy hitter task with $98 \%$ accuracy and 0.99 confidence level, we envision a compiler to generate a platform-agnostic sketch configuration (e.g., CountMin Sketch with $r \times d$ counters) that maintains errors $\leq 2 \%$ with 0.99 probability under any workload distribution.

\footnotetext{
${ }^{1}$ Device B runs in a CPU polling mode.
} 


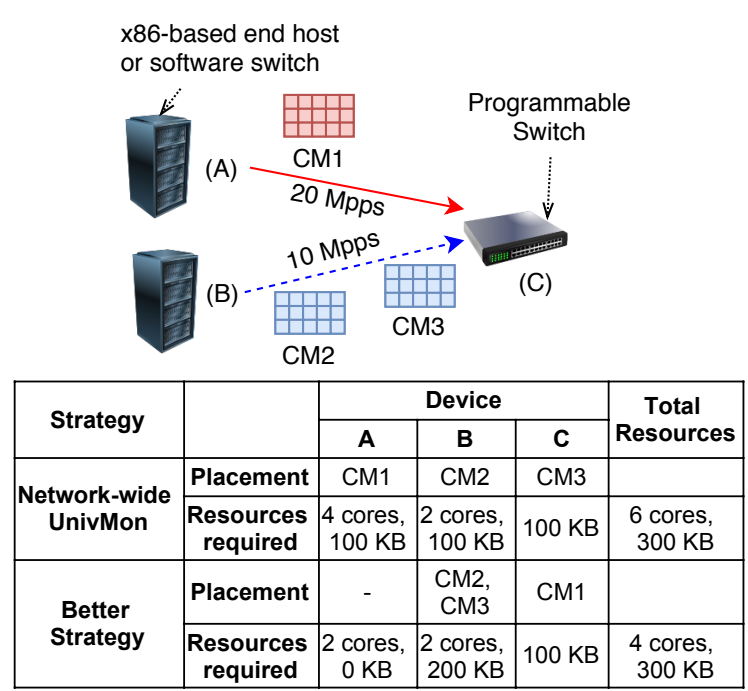

Figure 4: Example of network-wide UnivMon not optimally placing the sketches.

This is the first step towards network-wide device-aware resource management, which requires target-agnostic memory configurations treating the network-wide topology as a "one-big-switch" and corresponding performance characteristics on each hardware target as input.

Extension: [Expressiveness] If the network operator's telemetry queries $\mathcal{Q}$ cannot be compiled to $S$, can algorithm designers develop new sketching algorithms to address the failures?

While there have been significant advances in developing sketches for various telemetry tasks, the intents of network operators may still fall outside those of existing sketching algorithms. We need algorithm designers to step in and come up with improved or new sketches. Meanwhile, the theory community has already developed a rich pool of sketching tools that may be relevant to the operator's needs. The challenge lies in how to effectively collect and formulate these requirements to motivate algorithm designers to develop new algorithms or disprove the feasibility.

\subsection{Algorithm Designer \& Platform Vendor/De- veloper}

Problem 4: [Sketch Implementation] Given a sketch configuration $s \in S$ with device $d$, generate a sketch implementation $c_{s, d}$ to minimize the actual resource usages $r_{s, d}$ and $l_{s, d}$

Ideally, we want to generate optimized platform-specific sketch implementations for any sketch configuration and device instance. Today, this requires significant effort from both platform vendors/developers and algorithm designers to deliver optimized sketch implementation per hardware target [36, 45, 53, 58]. What is missing today are tools (e.g., optimizing compilers) to take as input an algorithm definition and configuration defined in a high-level language, and

\begin{tabular}{|l|c|c|c|c|}
\hline Resource & CountSketch & UnivMon & R-HHH & SketchLearn \\
\hline Match Crossbar & $10.0 \%$ & $177.2 \%$ & $476.9 \%$ & $347.7 \%$ \\
SRAM & $3.5 \%$ & $56.3 \%$ & $88.4 \%$ & $78.9 \%$ \\
Hash Bits & $3.7 \%$ & $59.6 \%$ & $91.6 \%$ & $82.1 \%$ \\
Hash Func. Calls & $62.5 \%$ & $1100.0 \%$ & $1562.5 \%$ & $700.0 \%$ \\
Stateful ALUs & $71.4 \%$ & $1142.9 \%$ & $1785.7 \%$ & $1600.0 \%$ \\
\hline
\end{tabular}

Table 2: Additional $\mathrm{H} / \mathrm{W}$ resource usage in Barefoot Tofino by existing sketch implementations. The numbers are normalized by the usage of baseline switch.p4

automatically output an implementation that is optimized for a particular hardware target. With such a tool, algorithm designers will not need to worry about how to implement a current or future sketching algorithm into the hardware architecture and platform developers will not worry about understanding the algorithmic details in order to implement the sketches. Existing efforts on P4 language and its targetspecific compilers are expected to contributing in this direction. Unfortunately, our benchmark demonstrates that existing sketch implementations on programmable switches using P4 are far from resource-efficient (Table 2 ${ }^{2}$. Compared to a fully functional switch implementation (switch.p4), existing sketches use excessive switch hardware resources (e.g., up to $15 \times$ more hash function calls and $17 \times$ more stateful ALUs).

Recent efforts have focused on performance bottlenecks of sketching algorithms run inside virtual software switches [36. 44. 59]. While they address the compute/memory bottlenecks in various software sketch implementations, their ideas do not directly transfer to other hardware platforms. For instance, NitroSketch [44] increases the memory footprint to reduce CPU consumption, but the key resource constraints in hardware context are different (e.g., processing stages, ALU, and hash function calls) [2]. SketchVisor [36] and ElasticSketch [59] split a sketch into a fast path and a slow path, and use the fast path to accelerate the packet processing. This type of idea is not particularly useful in hardware switches where all packet operations should stay in the fast path [17].

Extension: [Multi-Sketch Implementation] Given all sketch configurations $s \in S$ and device instance d, generate a consolidated sketch implementation $c_{d}$ for device $d$ such that the actual device resource usage $r_{d}$ and performance latency $l_{d}$ are minimized?

This extension is about optimizing the sketch implementation on a device when multiple sketch instances are present. Our observation is that many sketches share common primitive operations (hash computation, counter updates, etc.), and we expect that the actual resource usage and packet processing performance on a device $d^{*}$ can be further optimized to less than $\sum_{s} r_{s, d^{*}}$ and $\sum_{s} l_{s, d^{*}}$.

A recent proposal [32] shows the promises of using program synthesis to auto-generate fast processing hardware implementations on programmable switches using fewer hard-

\footnotetext{
${ }^{2}$ Sketch configurations in the table, R:rows, C:columns, and L:levels. CountSketch( $R=5, C=2048)$, UnivMon( $L=16, R=5$, $\mathrm{C}=2048)$, $\mathrm{R}-\mathrm{HHH}(\mathrm{L}=25, \mathrm{R}=5, \mathrm{C}=2048)$, SketchLearn( $\mathrm{L}=112$, $\mathrm{R}=1, \mathrm{C}=2048$ ).
} 
ware resources. While this direction is promising in general for Problem 4 and its extension, this work is a preliminary demonstration in one particular hardware architecture and we would like to see if a similar approach can be designed for other platforms and how many more resources it can save.

\subsection{Network Operator \& Platform Vendor/De- veloper}

Problem 5: [Sketch Configuration] Given a set of traffic workload characteristics $\mathcal{W}_{\nabla}$ and traffic-oblivious sketch configurations $S$ that meet accuracy requirements $\mathcal{R}_{\mathcal{A}}$, output a minimal platform-agnostic memory configuration for $\forall s \in S$ that meets the accuracy requirement

This problem entails finding a minimal memory configuration that meets a certain accuracy requirement for a sketch and a given type of traffic workload characteristics (e.g., skewness, number of flows). Problem 3 attempts to provide a traffic-oblivious memory configuration for the sketch to meet the accuracy requirement under any workloads. For platform vendors, it is of importance to fully understand the resourceaccuracy usage of the user functions running atop their platforms and to continue improving cost-efficiency of their architecture. In practice, network operators shall have basic understanding and expectation about the workloads such as skewness and distribution, and the traffic-oblivious configuration may not be tight anymore. For instance, Count Sketch can achieve better memory-accuracy tradeoff if the workload is skewed following some Zipfian distribution [21].

SketchLearn [37] leverages automated statistical inference to actively "learn" the traffic workload characteristics to configure its sketch on the fly, relieving the user burdens in the sketch memory configuration. While a learning-based approach is promising in resolving this problem, SketchLearn did not tackle the configurations of other types of sketches and we are unsure whether the model inference used in SketchLearn is an optimal choice.

Problem 6: [Verification] Given sketch implementation $c_{d}$ on device $d$, ensure that $c_{d}$ will correctly meet the accuracy requirements when running on $d$ ?

Once sketch implementations have been deployed to various devices, one question is that whether the on-device sketch instances will work as expected. Specifically, when an adversary is present, network operators want to verify the integrity of the sketch instances such that the output is correctly reflecting the network traffic conditions. We can think of this verification in two aspects: (1) Network operators can naturally verify the accuracy of sketches if the integrity of the on-device sketch instance is guaranteed. (2) If such integrity cannot be guaranteed, operators need to identify the occurrences when sketches failed to meet the accuracy requirements. Current platform vendors have been on an active race to offer secure enclave primitives on various hardware targets such as Intel SGX, AMD SEV, and ARM TrustZone for

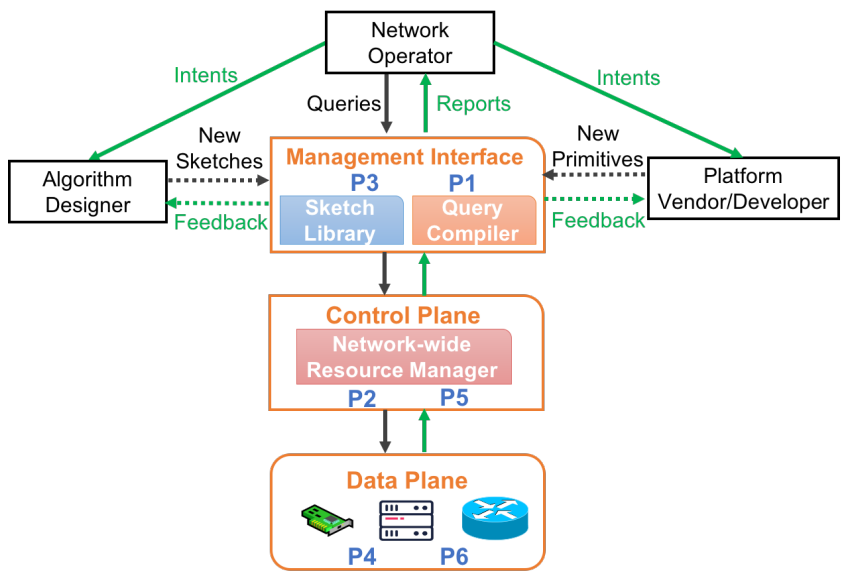

Figure 5: Sketch-based telemetry framework and stakeholder interactions.

mapping arbitrary functions to trusted memory. It remains an open challenge on how to leverage secure hardware capabilities as the "root-of-the-trust" for sketch-based telemetry.

Existing efforts [35, 51, 54] demonstrate the promises of protecting network functions with hardware enclaves (e.g., Intel SGX). However, those efforts are not capable of sketchbased telemetry because (1) sketches require high throughput guarantees while existing frameworks such as SafeBricks [51] and SGX-Box [35] incur high processing overhead, and (2) these efforts are designed for general-purpose network functions where redundant modules and complexities are included.

\section{A FUTURE ROADMAP}

We envision a sketch-based telemetry framework as depicted in Figure 5, assuming that research challenges P1-P6 described above and others have been properly addressed by the community. In this framework, we expect a management interface that has an expressive front-end/API to interact with network operators, algorithm designers, and platform vendors/developers. Some key components in the interface are 1) query compiler to translate operator intents into sketch configurations, and 2) sketch library to maintain stateof-the-art sketching definitions/implementations. In the control plane, there will be a network-wide resource manager taking input from the management interface and computing an optimized sketch placement and resource allocation based on the requirements. In the data plane, the optimized and verified sketch instances will be initialized across a network of heterogeneous devices based on the resource management decisions from the control plane.

We expect network operators, algorithm designers, and platform vendors/developers will have a way interacting with the telemetry framework as follows:

- Network operators: Operators can specify their telemetry needs via the management interface and receive the intended telemetry metrics via API. In the back-end, operator queries are translated to sketch configurations and their 
related device-level implementations to be deployed. In addition, operators can also describe their intents to cover some unsupported telemetry tasks.

- Algorithm designers: Algorithm designers can obtain new telemetry capability requests from operators and design new algorithms based on the requests. They can then add their new algorithms to the sketch ecosystem and get feedback about their implemented and evaluated algorithms in real-world scenarios.

- Platform vendors and developers: Platform vendors can receive new hardware capabilities requests, deliver new hardware capabilities, and update the device specifications accordingly. Platform developers can explore the sketch algorithm definitions and hardware capabilities in the sketching ecosystem, and deliver improved or new implementations to the sketch library.

Prior efforts have laid the groundwork for designing sketches and making them transition from a theoretical curiosity to a promising start for network telemetry. We hope that our vision, research challenges, and collaborative efforts from the stakeholders taken together can help transition sketch-based telemetry into "prime time" deployment.

\section{REFERENCES}

[1] Barefoot P4 Studio. https: / / www. barefootnetworks. com/products/brief-p4-studio/

[2] Barefoot Tofino.https: //barefootnetworks.com/products/brief-tofino/

[3] Broadcom Trident 3.https: //www.broadcom.com/ products/ethernet-connectivity/switching/ strataxgs/bcm56870-series/

[4] Data plane developer kit (dpdk). https: //software.intel.com/en-us/networking/dpdk

[5] Mellanox SmartNIC. https://www.mellanox.com/products/smartnic

[6] Netronome: Agilio SmartNICs and Software. https://fd.io/technology/

[7] P. K. Agarwal, G. Cormode, Z. Huang, J. Phillips, Z. Wei, and K. Yi. Mergeable summaries. In Proc. of ACM SIGMOD, 2012.

[8] N. Alon, Y. Matias, and M. Szegedy. The space complexity of approximating the frequency moments. In Proc. of ACM STOC, 1996.

[9] R. Alur, D. Fisman, and M. Raghothaman. Regular programming for quantitative properties of data streams. In European Symposium on Programming, 2016.

[10] AMD. Amd secure encrypted virtualization. https://developer.amd.com/sev/

[11] Apache. Apache druid.https://druid.apache.org/

[12] ARM TrustZone.https://developer.arm.com/ ip-products/security-ip/trustzone

[13] Z. Bar-Yossef, T. Jayram, R. Kumar, D. Sivakumar, and L. Trevisan. Counting distinct elements in a data stream. In RANDOM/APPROX. Springer, 2002.

[14] R. B. Basat, G. Einziger, R. Friedman, M. C. Luizelli, and E. Waisbard. Constant time updates in hierarchical heavy hitters. In Proc. of ACM SIGCOMM and CoRR/1707.06778, 2017.

[15] K. Borders, J. Springer, and M. Burnside. Chimera: A declarative language for streaming network traffic analysis. In Proc. of USENIX Security, 2012

[16] P. Bosshart, D. Daly, G. Gibb, M. Izzard, N. McKeown, J. Rexford, C. Schlesinger, D. Talayco, A. Vahdat, G. Varghese, et al. P4 Programming protocol-independent packet processors. ACM SIGCOMM CCR, 2014
[17] P. Bosshart, G. Gibb, H.-S. Kim, G. Varghese, N. McKeown, M. Izzard, F. Mujica, and M. Horowitz. Forwarding metamorphosis: Fast programmable match-action processing in hardware for sdn. In Proc. of ACM SIGCOMM, 2013.

[18] V. Braverman and R. Ostrovsky. Smooth histograms for sliding windows. In Proc. of IEEE FOCS, 2007.

[19] V. Braverman, R. Ostrovsky, and A. Roytman. Zero-one laws for sliding windows and universal sketches. In Proc. of APPROX/RANDOM, 2015

[20] S. Chandrasekaran, O. Cooper, A. Deshpande, M. J. Franklin, J. M. Hellerstein, W. Hong, S. Krishnamurthy, S. R. Madden, F. Reiss, and M. A. Shah. Telegraphcq: Continuous dataflow processing. In Proc. of ACM SIGMOD, 2003.

[21] M. Charikar, K. Chen, and M. Farach-Colton. Finding frequent items in data streams. In Proc. of ICALP, 2002.

[22] Cisco. Introduction to cisco ios netflow, 2012

[23] P. Clifford and I. Cosma. A simple sketching algorithm for entropy estimation over streaming data. In Artificial Intelligence and Statistics, 2013.

[24] G. Cormode. The continuous distributed monitoring model. ACM SIGMOD Record, 2013

[25] G. Cormode and S. Muthukrishnan. An Improved Data Stream Summary: The Count-Min Sketch and Its Applications. $J$. Algorithms, 2005.

[26] C. Cranor, T. Johnson, O. Spataschek, and V. Shkapenyuk. Gigascope: a stream database for network applications. In Proc. of ACM SIGMOD, 2003.

[27] M. Datar, A. Gionis, P. Indyk, and R. Motwani. Maintaining stream statistics over sliding windows. SIAM journal on computing, 2002.

[28] N. Duffield, C. Lund, and M. Thorup. Estimating flow distributions from sampled flow statistics. In Proceedings of the 2003 conference on Applications, technologies, architectures, and protocols for computer communications, pages 325-336, 2003.

[29] C. Estan and G. Varghese. New directions in traffic measurement and accounting. In Proc. of ACM SIGCOMM, 2002.

[30] P. Flajolet, ric Fusy, O. Gandouet, and et al. Hyperloglog: The analysis of a near-optimal cardinality estimation algorithm. In AOFA 2007.

[31] E. Gan, J. Ding, K. S. Tai, V. Sharan, and P. Bailis. Moment-based quantile sketches for efficient high cardinality aggregation queries. arXiv preprint arXiv:1803.01969, 2018.

[32] X. Gao, T. Kim, A. K. Varma, A. Sivaraman, and S. Narayana. Autogenerating fast packet-processing code using program synthesis In Proceedings of ACM HotNets, 2019.

[33] B. Ghazi, R. Panigrahy, and J. R. Wang. Recursive sketches for modular deep learning. In Proc. of ICML, 2019.

[34] A. Gupta, R. Harrison, M. Canini, N. Feamster, J. Rexford, and W. Willinger. Sonata: Query-driven streaming network telemetry. In Proc. of ACM SIGCOMM, 2018.

[35] J. Han, S. Kim, J. Ha, and D. Han. Sgx-box: Enabling visibility on encrypted traffic using a secure middlebox module. In Proceedings of the First Asia-Pacific Workshop on Networking, pages 99-105, 2017.

[36] Q. Huang, X. Jin, P. P. C. Lee, R. Li, L. Tang, Y.-C. Chen, and G. Zhang. Sketchvisor: Robust network measurement for software packet processing. In Proc. of ACM SIGCOMM, 2017.

[37] Q. Huang, P. P. Lee, and Y. Bao. Sketchlearn: Relieving user burdens in approximatemeasurement with automated statistical inference. In Proc. of ACM SIGCOMM, 2018.

[38] Intel SGX.https://software.intel.com/en-us/sgx

[39] N. Ivkin, R. B. Basat, Z. Liu, G. Einziger, R. Friedman, and V. Braverman. I know what you did last summer: Network monitoring using interval queries. POMACS (ACM SIGMETRICS), 2019.

[40] N. Ivkin, D. Rothchild, E. Ullah, I. Stoica, R. Arora, et al. Communication-efficient distributed sgd with sketching. In Proc. of NeurIPS, 2019.

[41] J. Jiang, F. Fu, T. Yang, and B. Cui. Sketchml: Accelerating distributed machine learning with data sketches. In Proc. of $A C M$ SIGMOD, 2018.

[42] B. Krishnamurthy, S. Sen, Y. Zhang, and Y. Chen. Sketch-based change detection: Methods, evaluation, and applications. In Proc. of 
ACM IMC, 2003.

[43] Y. Li, H. L. Nguyen, and D. P. Woodruff. Turnstile streaming algorithms might as well be linear sketches. In Proc. of ACM STOC, 2014.

[44] Z. Liu, R. Ben-Basat, G. Einziger, Y. Kassner, V. Braverman, R. Friedman, and V. Sekar. Nitrosketch: Robust and general sketch-based monitoring in software switches. In Proc. of ACM SIGCOMM. 2019.

[45] Z. Liu, A. Manousis, G. Vorsanger, V. Sekar, and V. Braverman. One sketch to rule them all: Rethinking network flow monitoring with univmon. In Proc. of ACM SIGCOMM, 2016.

[46] Z. Liu, S. Zhou, O. Rottenstreich, V. Braverman, and J. Rexford. Memory-efficient performance monitoring on programmable switches with lean algorithms. Proc. of SIAM/ACM APoCS, 2019.

[47] A. Metwally, D. Agrawal, and A. E. Abbadi. Efficient computation of frequent and top-k elements in data streams. In Proc. of ICDT, 2005.

[48] M. Moshref, M. Yu, R. Govindan, and A. Vahdat. Dream: dynamic resource allocation for software-defined measurement. In Proc. of ACM SIGCOMM, 2014.

[49] S. Narayana, A. Sivaraman, V. Nathan, P. Goyal, V. Arun, M. Alizadeh, V. Jeyakumar, and C. Kim. Language-directed hardware design for network performance monitoring. In Proc. of ACM SIGCOMM, 2017.

[50] G. Nychis, V. Sekar, D. G. Andersen, H. Kim, and H. Zhang. An empirical evaluation of entropy-based traffic anomaly detection. In ACM IMC, 2008.

[51] R. Poddar, C. Lan, R. A. Popa, and S. Ratnasamy. Safebricks: Shielding network functions in the cloud. In USENIX NSDI, 2018.

[52] R. Schweller, A. Gupta, E. Parsons, and Y. Chen. Reversible sketches for efficient and accurate change detection over network data streams In Proc. of ACM IMC, 2004.

[53] V. Sivaraman, S. Narayana, O. Rottenstreich, S. Muthukrishnan, and J. Rexford. Heavy-hitter detection entirely in the data plane. In Proc. of ACM SOSR, 2017.

[54] B. Trach, A. Krohmer, F. Gregor, S. Arnautov, P. Bhatotia, and C. Fetzer. Shieldbox: Secure middleboxes using shielded execution. In Proc. of ACM SOSR, 2018.

[55] J. A. Tropp, A. Yurtsever, M. Udell, and V. Cevher. Practical sketching algorithms for low-rank matrix approximation. SIAM Journal on Matrix Analysis and Applications, 2017.

[56] M. Wang, B. Li, and Z. Li. sflow: Towards resource-efficient and agile service federation in service overlay networks. In Proc. of IEEE ICDCS, 2004.

[57] D. P. Woodruff and Q. Zhang. Tight bounds for distributed functional monitoring. In Proc. of ACM STOC, 2012.

[58] M. Yang, J. Zhang, A. Gadre, Z. Liu, S. Kumar, and V. Sekar. Joltik: enabling energy-efficient" future-proof" analytics on low-power wide-area networks. In Proc. of ACM MobiCom, 2020.

[59] T. Yang, J. Jiang, P. Liu, Q. Huang, J. Gong, Y. Zhou, R. Miao, X. Li, and S. Uhlig. Elastic sketch: Adaptive and fast network-wide measurements. In Proc. of ACM SIGCOMM, 2018.

[60] M. Yu, L. Jose, and R. Miao. Software defined traffic measurement with opensketch. In Proc. of USENIX NSDI, 2013.

[61] Y. Yuan, D. Lin, A. Mishra, S. Marwaha, R. Alur, and B. T. Loo. Quantitative network monitoring with netqre. In Proc. of ACM SIGCOMM, 2017.

[62] Y. Zhou, Y. Zhang, C. Ma, S. Chen, and O. O. Odegbile. Generalized sketch families for network traffic measurement. POMACS (ACM SIGMETRICS), 2019. 